\title{
Validation of two real-time PCRs targeting the PE-PGRS 20 gene and the region of difference 4 for the characterization of Mycobacterium bovis isolates
}

\author{
M.L. Sales ${ }^{1}$, A.A. Fonseca Jr. ${ }^{1}$, L. Orzil ${ }^{1}$, A.P. Alencar ${ }^{1}$, M.A. Hodon ${ }^{1}$, \\ M.A. Issa ${ }^{1}$, P.M. Soares Filho ${ }^{1}$, M.R. Silva ${ }^{2}$, A.P. Lage ${ }^{3}$ and M.B. Heinemann ${ }^{3}$ \\ ${ }^{1}$ Lanagro/MG, Ministério da Agricultura, Pecuária e Abastecimento, \\ Pedro Leopoldo, MG, Brasil \\ ${ }^{2}$ EMBRAPA Gado de Leite, Juiz de Fora, MG, Brasil \\ ${ }^{3}$ Escola de Veterinária da Universidade Federal de Minas Gerais, \\ Belo Horizonte, MG, Brasil \\ Corresponding author: M.B. Heinemann \\ E-mail: mabryan@vet.ufmg.br \\ Genet. Mol. Res. 13 (2): 4607-4616 (2014) \\ Received June 12, 2013 \\ Accepted October 3, 2013 \\ Published June 18, 2014 \\ DOI http://dx.doi.org/10.4238/2014.June.18.3
}

\begin{abstract}
This study aimed to develop and validate real-time PCR for the diagnosis of Mycobacterium bovis isolates. Two hundred and seventy-four $M$. bovis isolates and $156 \mathrm{M}$. tuberculosis isolates were tested. Both qPCRs amplified all of the 274 M. bovis samples, but none of the 156 M. tuberculosis samples. The qPCR for PE-PGRS 20 had $91 \%$ efficiency and a detection limit of $0.32 \mathrm{ng}$ (sensitivity and specificity for qPCR "Mbovis.100" were 99.64 and 100\%, respectively). The qPCR for RD4 had 100\% efficiency, and a detection limit of 4 pg (diagnostic sensitivity and specificity were 100 and $100 \%$. The qPCR tests were performed using 4 extraction sets, 3 qPCR kits, and with a range of equipment; yet, all combinations produced similar results in a diagnostic
\end{abstract}


test, demonstrating the robustness of this method. The techniques proved to be efficient, robust, sensitive, and specific for the diagnosis of $M$. bovis.

Key words: Mycobacterium bovis; Real-time PCR; Validation; Bovine tuberculosis

\section{INTRODUCTION}

Bovine tuberculosis (TB) is a chronic granulomatous disease. The causative agent is Mycobacterium bovis, which is a member of the Mycobacterium tuberculosis complex (MTC) that infects several species of domestic animals and wildlife, in addition to human. Economic losses determined by this disease are manifested by a 10 to $20 \%$ reduction in milk production, in addition to weight gain, infertility, death, and condemnation of animal carcasses (Ministério da Agricultura Pecuária e Abastecimento, 2006). Yet, evidence supporting that $M$. bovis is a meat-borne hazard is lacking, worldwide. However, bovine TB has contributed to the limitation, and even prohibition, of Brazilian beef trade.

The tuberculin skin test is used for the traditional diagnosis of bovine TB, with bacterial isolation providing a confirmative and gold standard test [Ministério da Agricultura Pecuária e Abastecimento, 2006; OIE (World Organization for Animal Health), 2010]. A major limitation of M. bovis isolation is the delay in obtaining the results, because of the slow growth of the bacillus, requiring at least 4 weeks. After detecting the growth of the colonies, it is still necessary to confirm the species by phenotypic tests. Because large numbers of bacteria are required to perform all of the tests, it is often necessary to start a new culture of $M$. bovis, consequently delaying the final result by a further 3 weeks (Ministério da Agricultura Pecuária e Abastecimento, 2006).

Therefore, establishments suspected of bovine TB need diagnostic methods that are faster than bacteriological diagnosis, to clarify the actual status of the disease in their livestock herds. In addition, current phenotypic methods for TB bovine diagnosis are slow, and are not considered $100 \%$ reliable, because of the presence of intermediate strains that respond different to biochemical tests like niacin, the thiophene-2-carboxylic acid hydrazide variant $M$. tuberculosis, and the pyrazinamide variant M. bovis [Konno et al., 1967; OIE (World Organization for Animal Health), 2010]. The molecular approach might represent an alternative technique to replace or complement existing bacteriological methods of confirmation. The molecular approach is based on the detection of specific sequences of the $M$. bovis genome.

M. tuberculosis is the major cause of human TB; however, M. bovis may cause similar symptoms, and requires different treatment because of its natural resistance to pyrazinamide (Konno et al., 1967). The main mode of transmission of the agent from animals to man is by eating animal products, such as milk, or products contaminated with the bacillus (Silva et al., 2013). The situation is highly concerning in Brazil, where $41 \%$ of milk is produced illegally and consumed in the form of cheeses, yogurts, and milk, without proper thermal processing. This risk is further heightened by $5 \%$ of the herds and $0.85 \%$ of the cattle in Brazil being demonstrated to be tuberculin reactors by the Brazilian Ministry of Agriculture. Even the attenuated strain of $M$. bovis $\mathrm{BCG}$, which is commonly used in human vaccination, may cause clinical signs in humans, as already proven in patients with cellular immunodeficiency (Talbot et al., 1997) and neonates (Jou et al., 2009), and may generate complications exclusively related to BCG.

The similarity between the M. bovis and M. tuberculosis genomes presents a major 
problem in the use of molecular methods for the differentiation of these microorganisms. The genetic similarity between the two bacilli reaches $99.95 \%$, with the deletions being the main differences between the two (Fleischmann et al., 2002; Garnier et al., 2003). The accurate differentiation of MTC members is clinically important because of the intrinsic variations of these members to anti-tuberculosis drugs (Somoskovi et al., 2009). As such, the ability to differentiate among MTC members might potentially allow the surveillance of zoonotic exposure, in addition to providing accurate epidemiological information (Reddington et al., 2011).

Molecular biological methods have wide application in the laboratory diagnosis of animal diseases. PCR has several advantages compared to traditional methods of diagnosis, because it is highly sensitive, specific, and rapid. To produce a positive diagnosis, only a small number of cells are required; they do not have to be viable, and even poorly preserved samples may be used (Martinez et al., 2001). qPCR has higher precision, reproducibility, better quality control in the process, a lower risk of contamination, and eliminates the need for using electrophoresis (Wittwer et al., 2001). Furthermore, this technique reduces the analysis time to just 1-2 days, which is important to improve the currently time-consuming lengthy diagnosis of TB.

The current study aimed to develop 2 qPCR techniques using DNA intercalating agents for the molecular identification of $M$. bovis isolates.

\section{MATERIAL AND METHODS}

\section{Ethics statement}

We received M. tuberculosis samples from an already-existing collection at Empresa Brasileira de Pesquisa Agropecuária (Embrapa) Gado de Leite, Brazil. These samples were collected during a previous project conducted by Dr. Marcio Roberto Silva. All samples from humans were made anonymous. All M. bovis samples were isolated from lesions suggestive of tuberculosis that were collected from bovine in slaughterhouses around Brazil.

\section{Primer design and qPCR}

The primers designed to detect polymorphisms were based on the complete genome of M. bovis (Garnier et al., 2003). First, the sequences were subjected to BLAST ${ }^{\circledR}$ (http:// blast.ncbi.nlm.nih.gov/Blast.cgi) for verification of polymorphisms (Altschul et al., 1997). Then, the selected target mycobacterial sequences available on GenBank (NCBI, http://www. ncbi.nlm.nih.gov/) were aligned in BioEdit (Hall, 1999). After checking the ideal regions, namely those present only on M. bovis, the primers were designed in the program Primer3Plus (Untergasser et al., 2007), and analyzed using the program Oligo Analyser 3.1 (IDT, USA) for verification of secondary structures. In silico analytical specificity of the primers was tested with the PrimerBlast (NCBI, http://www.ncbi.nlm.nih.gov/tools/primer-blast/index. cgi?LINK_LOC=BlastHome) (Altschul et al., 1990).

The regions that were selected for the specific diagnosis of $M$. bovis were the gene coding for PE-PGRS 20 and the region of difference 4 (RD4). The primers used for PE-PGRS 20 were Mbovis.100.F: 5'-GGC TGT GAT GCT GCT TCC-3' (205009-205026 position in the genome, BX248337.1) and Mbovis.100.R: 5'-CTC CGT GCT AGG ACG GTT-3' (position 205108-205091), with a 100-bp segment being amplified. Primers for RD4 were Mbovis.88.F: 
5'-CGC CTT CCT AAC CAG AAT TG-3' (78694-78713 position in the genome, BX248337.1) and Mbovis.88.R: 5'-GGA GAG CGC CGT TGT AGG-3' (78764-78781 position), with an 88bp segment being amplified.

The best concentration for both qPCRs (Mbovis.100 and Mbovis.88) was defined as $0.375 \mathrm{nM}$ of each primer (IDT), $1.5 \mathrm{U}$ Hot Start GoTaq Polymerase (Promega, USA), $20 \%$ Colorless GoTaq Hot Start buffer 5X, $1.5 \mathrm{mM} \mathrm{MgCl}, 1 \mu \mathrm{L} 20 \mathrm{X}$ Eva Green (Biotium, Hayward, CA, USA), $2 \mu$ L Rox 10X (Biotium), $10 \mathrm{mM}$ dNTP made up to a final volume of $20 \mu \mathrm{L}$, and $2 \mu \mathrm{L}$ DNA. The PCR stages contained a step at $95^{\circ} \mathrm{C}$ for $5 \mathrm{~min}$, followed by 35 cycles at $95^{\circ} \mathrm{C}$ for $15 \mathrm{~s}, 63^{\circ} \mathrm{C}$ for $20 \mathrm{~s}$, and $72^{\circ} \mathrm{C}$ for $30 \mathrm{~s}$, with the reading cycle length. The curve denaturation was performed at $72^{\circ} \mathrm{C}$ to $99^{\circ} \mathrm{C}$, with intervals of fluorescence at every $1 \%$ rise in temperature.

Real-time PCRs (qPCR) were performed in ABI7500 (Applied Biosystems, USA). Tests were performed using trial and error adjustments, in accordance with primer concentrations and annealing temperature, to adjust reagent concentrations. Samples were only considered positive when the curve analysis showed melting temperature peaks that did not differ by more than $1^{\circ} \mathrm{C}$ from the positive control used in the same experiment. All tests were performed with positive and negative controls and blank tubes to detect the reagent contamination.

\section{Samples}

Standard strains of $M$. bovis AN5 México CRNC 36, $M$. bovis AN5 CRNC 02, $M$. bovis AN5 CRNC 01, and M. bovis BCG CRNC were used as positive controls during all tests in this study. Standard strains of M. tuberculosis H37Rv CRNC 23 and M. tuberculosis H37Ra CRNC 25 were used as negative controls.

The following strains were used in analytical specificity tests: $M$. fortuitum CRNC 10, M. kansasii CRNC 48, M. kansasii CRNC 18, M. gordonae CRNC 16, M. avium D4 CRNC 05, M. avium paratuberculosis CRNC 26, M. avium 1500 CRNC 15, M. intracellulare CRNC 17, M. avium 2045 CRNC 14, M. marinum CRNC 19, M. scrofulaceum CRNC 49, $M$. scrofulaceum CRNC 20, M. szulgai CRNC 21, M. triviale CRNC 22, M. fortuitum peregrinum CRNC 11, M. phei CRNC 12, Rhodococcus equi CRC 09/01, Corynebacterium pseudotuberculosis CRC 09/02, and Nocardia asteroides CRC 10/01.

Four hundred and thirty (274 M. bovis and 156 M. tuberculosis) isolates from Brazil were used to test the diagnostic sensitivity and specificity. All of the samples that were tested were previously characterized biochemically. All M. tuberculosis samples were previously characterized by amplification of the pncA gene (Scorpio and Zhang, 1996), and its polymorphism was detected by the cleavage of the amplicon with Eco065I, as previously described (Barouni et al., 2004). This analysis was carried out at the Laboratory of Molecular Biology Applied to Mycobacteria, Oswaldo Cruz Institute, Fiocruz, Brazil.

\section{Phenotype-based speciation methods}

All positive cultures were subjected to additional phenotypic speciation methods, including biochemical tests to distinguish mycobacterial strains. Tests included catalase at room temperature and at $68^{\circ} \mathrm{C}$. In addition niacin, nitrate, pyrazinamidase, urease, and drug susceptibility testing were conducted (Kent and Kubica, 1985). 


\section{Validation criteria}

The parameters for the validation methodology were performed using parameters described in the Manual of Diagnostic Tests and Vaccines for Terrestrial Animals [OIE (World Organization for Animal Health), 2010] and as proposed by Fonseca Jr. et al. (2013).

\section{Reaction efficiency}

The reaction efficiency was tested by diluting DNA samples extracted from M. bovis AN5 México CRNC 36 (Canevari Castelão et al., 2014). Each dilution was tested in duplicate, and the concentration was estimated by the spectrophotometer UV light Nanovue ${ }^{\circledR}$ (GE Healthcare, Salt Lake City, UT, USA). Standard curves for both qPCRs were created to determine the optimal concentration of primers, which were defined as $\mathrm{R}^{2}$ minimum of 0.99 and a minimum efficiency of $90 \%$.

\section{Limit of detection (LOD)}

LOD was determined by the dilution of the standard strain in base ten. The dilutions were tested in triplicate, and the LOD was determined as the dilution at which all replicates were positive. The LOD was confirmed by repeating the qPCR 21 times.

\section{Repeatability and reproducibility}

The repeatability was estimated using 7 samples of $M$. bovis. We extracted DNA from 7 samples during 3 days of sampling, and submitted the extracted nucleic acid to qPCR in triplicate. To assess possible changes in the test, a second analyst performed the technique using the same criteria and the same samples.

Twenty-one samples were sent to another laboratory to assess interlaboratory reproducibility. Sampling was performed according to OIE (World Organization for Animal Health, 2010), using bacterial suspensions of M. bovis, M. tuberculosis, and mycobacteria other than M. tuberculosis.

\section{Robustness}

The following variables were analyzed as possible alternatives for the diagnosis of bovine TB. Four extraction kits were tested: Nucleospin Triprep ${ }^{\circledR}$ (Macherey Nagel, Bethlehem, PA, USA) DNeasy Blood \& Tissue Kit ${ }^{\mathbb{B}}$ (Qiagen, Valencia, CA, USA), AccuPrep Genomic DNA Extraction $\mathrm{Kit}^{\mathbb{B}}$ (Bioneer, Alameda, CA, USA), and Genomic DNA Isolation $\mathrm{Kit}^{\mathbb{\circledR}}$ (RBC, New Taipei City, Taiwan), in addition to boiling for $30 \mathrm{~min}$ at $90^{\circ} \mathrm{C}$. The following qPCR kits were also tested: Fast qPCR Master Mix Plus EvaGreen ${ }^{\circledR}$ (Biotium), 2.0X RealQ-PCR Master Mix ${ }^{\circledR}$ with Sybr Green (Ampliqon, Odense, Denmark), and Platinum SYBR Green qPCR Supermix-UDG ${ }^{\circledR}$ (Invitrogen, Carlsbad, CA, USA). The 7 samples previously submitted to the qPCR technique were tested by all these kits in triplicate in the termocycler ABI7500 (Applied Biosystems). The qPCRs were also tested in the thermocycler Rotorgene 3000 (Qiagen). 


\section{Statistical analysis}

We used the McNemar test to compare paired proportions, the kappa test to determine the measure of agreement between two tests classifying each sample, and the sensitivity and specificity analysis.

\section{Sensitivity and specificity}

For the sensitivity and specificity analysis, $274 \mathrm{M}$. bovis and $156 \mathrm{M}$. tuberculosis isolates from Brazil were used. Each sample was simultaneously tested by phenotypic and qPCR methods (Mbovis.100 and Mbovis.88). The qPCR method was compared with the phenotypebased speciation method, as a gold standard.

\section{RESULTS}

qPCR

The efficiency of qPCR Mbovis. 88 and qPCR Mbovis. 100 was 100 and $90.96 \%$, respectively, with both having an $\mathrm{R}^{2}$ above 0.99 . Melting peaks were $92.20^{\circ} \mathrm{C}$ for qPCR Mbovis. 100 (standard deviation $0.37^{\circ} \mathrm{C}$ ) and $82.75^{\circ} \mathrm{C}$ for qPCR Mbovis. 88 (standard deviation $\left.0.30^{\circ} \mathrm{C}\right)$.

LOD was calculated based on serial dilutions of M. bovis DNA. As indicated by the reaction efficiency, qPCR Mbovis.88 could detect lower quantities of bacterial DNA (4 pg) compared to qPCR Mbovis.100 (0.32 ng).

\section{Repeatability, reproducibility, and robustness}

The average values and standard deviations of $\mathrm{Ct}$ for each day in the repeatability and intralaboratory reproducibility tests for qPCR Mbovis. 100 and qPCR Mbovis. 88 are shown in Tables 1 and 2, respectively. The results indicate that both qPCRs may be reproduced in the laboratory without major differences in qualitative results. Reproducibility was $100 \%$ in the interlaboratory trials.

All qPCR kits and variations in DNA extraction produced similar results, with low standard deviations. The peaks in the melting curve analysis differed in each kit. Table 3 shows variations in the denaturation temperature for qPCR Mbovis.100. Denaturation temperatures were used for PCR kits "Fast-Plus EvaGreen qPCR Master Mix" (Biotium), and "2.0X RealQ-PCR with Sybr Green Master Mix" (Neobio, Brazil) and Rotor gene 3000 (Qiagen).

\section{Statistical analyses}

The McNemar test showed no significant difference between both qPCR and the phenotypic tests. The Kappa test showed perfect agreement between the the two qPCRs and the phenotypic tests. 
Table 1. Mean cycle threshold $(\mathrm{Ct})$ values and standard deviation $(\sigma)$ for each day of test $(\mathrm{Ct} 1, \mathrm{Ct} 2, \mathrm{Ct} 3)$ for qPCR Mbovis. 100 repeatability and reproducibility.

\begin{tabular}{|c|c|c|c|c|c|c|c|c|c|c|c|c|}
\hline \multirow[t]{2}{*}{ Sample } & \multicolumn{6}{|c|}{ First analyst } & \multicolumn{6}{|c|}{ Second analyst } \\
\hline & $\mathrm{Ct} 1$ & $\sigma$ & $\mathrm{Ct} 2$ & $\sigma$ & $\mathrm{Ct} 3$ & $\sigma$ & Ct1 & $\sigma$ & $\mathrm{Ct} 2$ & $\sigma$ & $\mathrm{Ct} 3$ & $\Sigma$ \\
\hline 1 & 22.41 & 0.28 & 18.94 & 0.03 & 16.08 & 0.12 & 17.92 & 0.05 & 22.15 & 0.17 & 18.13 & 0.60 \\
\hline 2 & 21.13 & 0.01 & 20.48 & 0.08 & 17.55 & 0.07 & 20.34 & 0.10 & 23.66 & 0.12 & 18.80 & 0.95 \\
\hline 3 & 21.37 & 0.08 & 24.31 & 0.09 & 21.89 & 0.10 & 20.05 & 0.07 & 24.15 & 0.12 & 22.63 & 0.07 \\
\hline 4 & 23.98 & 0.26 & 24.73 & 0.07 & 18.49 & 0.11 & 24.57 & 0.22 & 24.40 & 0.07 & 26.86 & 0.12 \\
\hline 5 & 20.19 & 0.08 & 22.47 & 0.11 & 20.26 & 0.16 & 20.54 & 0.07 & 27.02 & 0.74 & 25.18 & 0.05 \\
\hline 6 & 22.92 & 0.04 & 20.57 & 0.03 & 19.84 & 0.28 & 21.18 & 0.20 & 23.79 & 0.46 & 22.56 & 1.36 \\
\hline 7 & 25.89 & 0.12 & 21.86 & 0.04 & 21.05 & 0.05 & 23.24 & 0.11 & 26.74 & 0.14 & 20.41 & 0.09 \\
\hline $\mathrm{PC}$ & 25.64 & - & 29.02 & - & 26.91 & - & 25.86 & - & 29.69 & - & 28.30 & \\
\hline
\end{tabular}

$\mathrm{PC}=$ positive control.

Table 2. Mean cycle threshold $(\mathrm{Ct})$ values and standard deviation $(\sigma)$ for each day of test $(\mathrm{Ct} 1, \mathrm{Ct} 2, \mathrm{Ct} 3)$ for qPCR-88 repeatability and reproducibility.

\begin{tabular}{|c|c|c|c|c|c|c|c|c|c|c|c|c|}
\hline \multirow[t]{2}{*}{ Sample } & \multicolumn{6}{|c|}{ First analyst } & \multicolumn{6}{|c|}{ Second analyst } \\
\hline & Ct1 & $\sigma$ & $\mathrm{Ct} 2$ & $\sigma$ & Ct3 & $\sigma$ & Ct1 & $\sigma$ & $\mathrm{Ct} 2$ & $\sigma$ & $\mathrm{Ct} 3$ & $\sigma$ \\
\hline 1 & 21.44 & 0.24 & 18.52 & 0.15 & 17.45 & 0.14 & 19.30 & 0.43 & 19.58 & 0.29 & 18.48 & 0.11 \\
\hline 2 & 20.78 & 1.10 & 16.28 & 0.09 & 15.15 & 0.87 & 17.91 & 0.07 & 16.67 & 0.45 & 16.86 & 0.13 \\
\hline 3 & 24.37 & 0.15 & 21.85 & 0.23 & 20.25 & 0.13 & 25.22 & 0.32 & 25.45 & 0.80 & 24.42 & 0.61 \\
\hline 4 & 22.96 & 0.23 & 20.45 & 0.38 & 19.23 & 0.23 & 21.77 & 0.22 & 22.20 & 0.27 & 21.13 & 0.20 \\
\hline 5 & 25.89 & 0.28 & 23.19 & 0.49 & 21.35 & 0.33 & 27.26 & 0.35 & 27.50 & 0.11 & 25.81 & 0.08 \\
\hline 6 & 27.98 & 0.54 & 24.94 & 0.09 & 22.26 & 0.22 & 31.60 & 0.32 & 31.87 & 0.57 & 29.10 & 0.12 \\
\hline 7 & 23.62 & 0.07 & 20.64 & 0.14 & 19.06 & 0.24 & 22.19 & 0.31 & 22.64 & 0.34 & 21.49 & 0.31 \\
\hline $\mathrm{PC}$ & 22.79 & - & 19.84 & - & 19.00 & - & 20.13 & - & 21.23 & - & 19.48 & - \\
\hline
\end{tabular}

$\mathrm{PC}=$ positive control.

Table 3. Variation of melting curve peaks in robustness tests.

\begin{tabular}{|c|c|c|c|c|c|c|c|c|}
\hline \multirow[t]{2}{*}{ Sample } & \multicolumn{8}{|c|}{ Melting peak uncertainty } \\
\hline & 1 & 2 & 3 & 4 & 5 & 6 & 7 & 8 \\
\hline Mean & 91.87 & 92.12 & 91.93 & 92.46 & 89.48 & 93.43 & 91.45 & 95.55 \\
\hline$\sigma$ & 0.22 & 0.37 & 0.30 & 0.52 & 0.57 & 0.37 & 0.34 & 0.80 \\
\hline Maximum & 92.09 & 92.49 & 92.30 & 92.48 & 90.06 & 93.81 & 91.79 & 96.36 \\
\hline Minimum & 91.66 & 91.74 & 91.55 & 91.95 & 88.91 & 93.06 & 91.11 & 94.74 \\
\hline
\end{tabular}

DNA extraction kits: 1 = AccuPrep Genomic DNA Extraction Kit (Bioneer); 2 = DNeasy Blood \& Tissue Kit (Qiagen); 3 = Nucleospin Triprep; 4 = Genomic DNA Isolation Kit (Real Biotech Corporation). PCR kits: 5 = "FastPlus EvaGreen qPCR Master Mix" (Biotium); 6 = "2.0X RealQ-PCR Master Mix com Sybr Green" (Neobio); 7 = Platinum SYBR Green qPCR Super Mix-UDG (Invitrogen); 8 = Equipament Rotor Gene 3000 (Qiagen).

\section{Sensitivity and specificity}

Neither qPCR amplified any reference sample from the genus Mycobacterium that was used in this study, besides $M$. bovis. Only one $M$. bovis isolate that was tested in the sensitivity test was not positive in qPCR Mbovis.100; however, all M. bovis isolates were positive in qPCR Mbovis.88. The DNA extracted from M. tuberculosis was not amplified in any qPCR. The diagnostic sensitivity and specificity for qPCR Mbovis. 100 was $99.64 \%(95 \% \mathrm{CI}=97.98-99.94 \%)$ and $100 \%(95 \% \mathrm{CI}=98.02-100 \%)$, respectively. The diagnostic sensitivity and specificity for qPCR Mbovis. 88 was $100 \%(95 \% \mathrm{CI}=98.65-100 \%)$ and $100 \%(95 \% \mathrm{CI}=98.02-100 \%)$, respectively. 


\section{DISCUSSION}

Both qPCR showed a high degree of diagnostic sensitivity and specificity compared to phenotypic analysis, demonstrating that the selected regions are useful for the differentiation of M. bovis and M. tuberculosis. The DNA sequence detected by qPCR Mbovis. 88 (targeting RD4 in M. bovis genome) was only present in strains derived from M. bovis, and did not exist in the $M$. tuberculosis complex, even in those most genetically similar species like $M$. caprae (Garnier et al., 2003; Sales et al., 2014). Because of the MTC clonal profile and the low rate of recombination, a reversal in this deleted region is unlikely. The region detected by qPCR Mbovis. 100 is a gene coding for 1 protein of the PE-PGRS family. Genetic variation occurs in the genes that code for PE-PGRS M. bovis and M. tuberculosis because of insertions and deletions (Sreenu et al., 2006). These genetic factors, combined with the large sample size used in this study, increase the confidence in using these qPCRs for the identification of M. bovis.

The repeatability and reproducibility of a technique must be scientifically tested to ensure that the same diagnosis and identification of microorganisms is consistently obtained under different conditions. DNA extraction was responsible for differences in $\mathrm{Ct}$ values between days, analysts, and qPCR. Both qPCRs were developed for qualitative results; hence, the amount of DNA added to reaction mixes was not precise, varying between 20 and $200 \mathrm{ng}$, explaining the data shown in both tables. These two parameters must also be confirmed in the regulation for laboratory accreditation (Freitas et al., 2006). Hence, qPCR validation was essential to prove that the same results could be reproduced despite variations in day, analyst, reagents, equipment, and laboratory.

Ring tests are part of the requirements for the validation of a diagnostic test [OIE (World Organization for Animal Health), 2010]; hence, the validation phases of a method should not only include tests within a laboratory, but proof of performance at different institutions as is known that PCR results may be slightly different in different laboratories, because of differences in equipment, reagents or the implementation procedures by different analysts (Eischeid, 2011). The interlaboratory tests conducted in this study produced $100 \%$ replicability. There were few variations in technique, because both laboratories use the same reagents and equipment for PCR; however, the results confirmed that both qPCRs could be performed safely in other laboratories.

To evaluate the performance of qPCR, we assessed different qPCR equipment, kit DNA extraction kits, and amplification kits. All resulting samples produced positive results, which were amplified with specific denaturation peaks when compared to the positive control.

The peaks in the melting curve analysis results were quite variable in all tests; however, these changes were expected. The Rotorgene 3000 has a different fluorescence detection and heat exchange system to ABI7500, which generates discrepant peaks in melting curves; however, this variation is minimal within the same equipment. Extraction kits generate different residues during DNA extraction, including ions that influence DNA dissociation.

PCR mixes produced by different companies have buffers with factors causing differences at the specific point that the denaturation of the double-stranded DNA occurs (Wittwer et al., 2001). One of the kits used in this study had even had a different intercalating agent, SYBR Green, which has different saturation DNA molecule characteristics compared to Eva Green (Eischeid, 2011). Yet, the results remained consistent across all kits, confirming the robustness of the qPCRs. None of these factors invalidated the results or prevented the use of these reagents. 
The target regions for both of the qPCRs used in this study were based on long polymorphisms, which reduced the possibility of false-positive results. Besides the fact that a large number of samples were used to validate these techniques, long polymorphisms offer several advantages over other PCR techniques that rely only on single nucleotide polymorphisms (Goh et al., 2006), require electrophoresis on agarose gels (Shah et al., 2002), or use a small amount of samples (Bakshi et al., 2005). The methodologies tested in this study proved to be reliable, despite the use of different reagents and equipment. These variables have not been subject to extensive testing in any of the previously published studies, despite representing common differences in molecular biology laboratories.

Both of the qPCRs analyzed in the present study have potential application as a rapid method for the diagnosis of bovine $\mathrm{TB}$, and might also have public health utility, particularly in areas (or populations) where M. bovis is prevalent. Furthermore, these qPCRs could be used to obtain estimates of the prevalence or incidence rates of bovine and human infections caused by M. bovis. This strategy could be expanded beyond Brazil, for epidemiological purposes in areas such as Africa, Southeast Asia, Central America, South America, and other regions where M. bovis is transmitted from animals to humans (and vice-versa), or is a suspected problem.

\section{ACKNOWLEDGMENTS}

We thank CNPq (\#410595/2006-3), INCT-Pecuária, and the Brazilian Ministry of Agriculture for providing financial support.

\section{REFERENCES}

Altschul SF, Gish W, Miller W, Myers EW, et al. (1990). Basic local alignment search tool. J. Mol. Biol. 215: 403-410.

Altschul SF, Madden TL, Schaffer AA, Zhang J, et al. (1997). Gapped BLAST and PSI-BLAST: a new generation of protein database search programs. Nucleic Acids Res. 25: 3389-3402.

Bakshi CS, Shah DH, Verma R, Singh RK, et al. (2005). Rapid differentiation of Mycobacterium bovis and Mycobacterium tuberculosis based on a 12.7-kb fragment by a single tube multiplex-PCR. Vet. Microbiol. 109: 211-216.

Barouni AS, Augusto CJ, Lopes MT, Zanini MS, et al. (2004). A pncA polymorphism to differentiate between Mycobacterium bovis and Mycobacterium tuberculosis. Mol. Cell Probes 18: 167-170.

Canevari Castelão AB, Nishibe C, Moura A, de Alencar AP, et al. (2014). Draft genome sequence of Mycobacterium bovis strain AN5, used for production of purified protein derivative. Genome Announc. 2: e00277-14.

Eischeid AC (2011). SYTO dyes and EvaGreen outperform SYBR Green in real-time PCR. BMC Res. Notes 4: 263.

Fleischmann RD, Alland D, Eisen JA, Carpenter L, et al. (2002). Whole-genome comparison of Mycobacterium tuberculosis clinical and laboratory strains. J. Bacteriol. 184: 5479-5490.

Fonseca Jr AA, Cottorello AC, Dias NL, D’Ambros R, et al. (2013). PCR em tempo real para detecção do vírus da doença de Aujeszky. Arq. Bras. Med. Vet. Zootec. 65: 801-808.

Freitas EI, Lemos AA and Marin VA (2006). Validação de métodos alternativos qualitativos na detecção de patógenos alimentares. Ciênc. Saúde Coletiva 11: 1073-1083.

Garnier T, Eiglmeier K, Camus JC, Medina N, et al. (2003). The complete genome sequence of Mycobacterium bovis. Proc. Natl. Acad. Sci. U. S. A. 100: 7877-7882.

Goh KS, Fabre M, Huard RC, Schmid S, et al. (2006). Study of the gyrB gene polymorphism as a tool to differentiate among Mycobacterium tuberculosis complex subspecies further underlines the older evolutionary age of 'Mycobacterium canettii'. Mol. Cell Probes 20: 182-190.

Hall TA (1999). BioEdit: a user-friendly biological sequence alignment editor and analysis program for Windows 95/98/ NT. Nucleic Acids Symp. Ser. 41: 95-98.

Jou R, Huang WL and Su WJ (2009). Tokyo-172 BCG vaccination complications, Taiwan. Emerg. Infect. Dis. 15: 15251526. 
Kent PT and Kubica GP (1985). Public Health Mycobacteriology: A Guide for the Level III Laboratory. Centers for Disease Control and Prevention, Atlanta.

Konno K, Feldmann FM and McDermott W (1967). Pyrazinamide susceptibility and amidase activity of tubercle bacilli. Am. Rev. Respir. Dis. 95: 461-469.

Martinez G, Harel J and Gottschalk M (2001). Specific detection by PCR of Streptococcus agalactiae in milk. Can. J. Vet. Res. 65: 68-72.

Ministério da Agricultura Pecuária e Abastecimento (2006). Programa Nacional de Controle e Erradicação da Brucelose e Tuberculose - PNCEBT. Ministério da Agricultura, Pecuária e Abastecimento, Brasília.

OIE (World Organization for Animal Health) (2010). Principles and Methods of Validation of Diagnostic Assays for Infectious Diseases. In: Terrestrial Manual. Paris Office International des Epizooties, Paris.

Reddington K, O'Grady J, Dorai-Raj S, Niemann S, et al. (2011). A novel multiplex real-time PCR for the identification of mycobacteria associated with zoonotic tuberculosis. PLoS One 6: e23481.

Sales ML, Fonseca AA Jr, Sales EB, Cottorello AC, et al. (2014). Evaluation of molecular markers for the diagnosis of Mycobacterium bovis. Folia Microbiol. Doi: 10.1007/s12223-014-0317-3. [Epub ahead of print].

Scorpio A and Zhang Y (1996). Mutations in pncA, a gene encoding pyrazinamidase/nicotinamidase, cause resistance to the antituberculous drug pyrazinamide in tubercle bacillus. Nat. Med. 2: 662-667.

Shah DH, Verma R, Bakshi CS and Singh RK (2002). A multiplex-PCR for the differentiation of Mycobacterium bovis and Mycobacterium tuberculosis. FEMS Microbiol. Lett. 214: 39-43.

Silva MR, Rocha AS, da Costa RR, de Alencar AP, et al. (2013). Tuberculosis patients co-infected with Mycobacterium bovis and Mycobacterium tuberculosis in an urban area of Brazil. Mem. Inst. Oswaldo Cruz, 108.

Somoskovi A, Dormandy J, Mayrer AR, Carter M, et al. (2009). "Mycobacterium canettii" isolated from a human immunodeficiency virus-positive patient: first case recognized in the United States. J. Clin. Microbiol. 47: 255-257.

Sreenu VB, Kumar P, Nagaraju J and Nagarajaram HA (2006). Microsatellite polymorphism across the M. tuberculosis and M. bovis genomes: implications on genome evolution and plasticity. BMC Genomics 7: 78.

Talbot EA, Williams DL and Frothingham R (1997). PCR identification of Mycobacterium bovis BCG. J. Clin. Microbiol. 35: 566-569.

Untergasser A, Nijveen H, Rao X, Bisseling T, et al. (2007). Primer3Plus, an enhanced web interface to Primer3. Nucleic Acids Res. 35: W71-W74.

Wittwer CT, Herrmann MG, Gundry CN and Elenitoba-Johnson KS (2001). Real-time multiplex PCR assays. Methods 25: $430-442$. 